\title{
EREBEA
}

\author{
Revista de Humanidades \\ y Ciencias Sociales \\ Núm. 8 (2018), pp. 61-87 \\ ISSN: 0214-0691
}

\section{NOMBRES TRADICIONALES KAMBA COMO INSTRUMENTO DE RELACIÓN Y ELEMENTO REPRESENTATIVO DE IDENTIDAD Y PATRIMONIO INTANGIBLE ENTRE LOS AKAMBA DEL ÁREA RURAL MaChaKos \\ David Caballero Mariscal Universidad de Granada}

RESUMEN

Los kamba suponen uno de los colectivos más extendidos en Kenia, teniendo en cuenta las controversias que puede generar la conceptualización de grupo étnico, o su categoría más colonial etnocéntrica, tribu. Uno de los aspectos más significativos que caracterizan a los kamba de la región rural del interior del condado de Machakos es que a la persona al nacer se le otorga un nombre tradicional relacionado con el momento del día en el que nace, el tiempo atmosférico o cualquier otra circunstancia relevante que implique la gestación o el parto. Este hecho proyecta, en cierta medida, un modo de relación y un rol de la persona en el contexto familiar y social.

\section{Palabras Clave}

kamba; nombres kikamba; tradición; familia; relaciones; identidad patrimonio inmaterial.

Fecha de recepción: 22 de julio de 2018 Fecha de aceptación: 1 de oct. de 2018
ABSTRACT

Kamba people is one of the most extended collectives in Kenya, if we take into account the controversies that can result from the conceptualization of ethnic group, of its colonial-ethnocentric category: tribe. One of the most striking features of the kamba living in the rural areas of the Machakos Count is the issue of the kamba name given at birth, traditionally related to the moment of the day, the weather, or another different circumstance concerning to the pregnancy or childbirth. This fact is projected, to a greater or a lesser extent, in the way of relating, and, in turn, in the role of the person both in the social and in the family contexts.

\section{KeYwords}

kamba; kikamba names; tradition; familiy; relations; identity; immaterial heritage. 


\section{INTRODUCCIÓN}

Derivado de la experiencia de convivencia y trabajo de campo con una familia kamba durante 2017, surgió este proyecto, que trata de aunar la observación directa y participante con la elaboración de una interpretación sobre la experiencia vivida en el contacto y la constante interacción con los miembros de la familia nuclear, la extensa, parte del clan y la comunidad más inmediata. No obstante, desde este proceso de relaciones, marcadas por un constante e intenso proceso adaptativo, y por ende, cargado de contradicciones e incomprensiones mutuas, propias de todo acoplamiento a un grupo humano con el que no se han tenido contactos anteriores, surgieron distintos interrogantes. El primero de éstos se correspondía con la pregunta clave, y que podría sonar a perogrullada: ¿Qué es un akamba? ¿Qué es kamba? ¿Qué kikamba? La segunda de las cuestiones, ante la dificultad de responder a la primera, y la osadía de haber tratado de responder, con una serie de categorías de cierta maagnitud, pero caducas, y lastradas de prejuicios, fue la de ¿Qué puedo decir de los akamba, de sus relaciones familiares? ¿Qué elementos implican sus señas de identidad y constituyen, por tanto, su, patrimonio inmaterial, recibido y transmitido de generación a generación y que da sentido de grupo y contribuye a su cohesión?

Es dificultoso, en esta dirección, marcar el punto de equilibrio entre la comprensión del dinamismo de un colectivo como los kamba, con la gran diversidad que estos representan, y la variedad dentro de unos parámetros; y la huida de un esencialismo que permite ahondar en categorías que facilitan la comprensión de un corpus de normas y comportamientos en general que nos resultan ajenos. Bajo la justificación de ser un mecanismo casi a modo de estrategia de comprensión, el hecho subsumir una amplitud de realidades a la unidad de un proyecto, no deja sino entrever la falacia de "la invención de la tradición"1. Por supuesto, la intencionalidad intrínseca, carente de intencionalidad banal, es la expresada por Díaz de Rada, quien manifiesta que llevar a cabo una etnografía implicaría "entre otras cosas, en el alcanzar una comprensión lo más detallada posible de las preguntas que parecen formularse una y otra vez los propios protagonistas de la vida

1 G. Celigueta; G. Orobitg; y P. Pitarch (coord.), Aprender ciudadania en Guatemala. Modernidad indigena, 'indigeneidad' e innovación social desde la perspectiva del género. Barcelona: Ube, 2014, p. 142 . 
social" ${ }^{2}$. En este sentido, observar y participar de los modos de interacción, de las costumbres, de creencias y tradiciones, desde esa perspectiva, precisamente, aquélla que trata de retratar, aún sabiendo que sólo se podrán poner en relieve elementos parciales de una identidad en constante proceso de cambio, de transformación y de cierne. Y a pesar de que "la búsqueda de la totalidad, el holismo, es una de las intenciones básicas de la investigación etnográfica" (Díaz de Rada, 2003, p. 237), no pretendemos, en este caso caminar hacia un "holismo etnográfico" que trate de "formular enunciados de universalidad ilimitada sino representar los diferentes elementos culturales mediante el establecimiento de relacionessistemáticas entre ellos"3 (Molina, Tomé y Valencia, 2009, p. 25).

Desde la observación de los distintos aspectos que se han observado, se pretende describir cómo el nombre tradicional en lengua kamba, elemento significativo de su patrimonio inmaterial junto a la lengua en sí y determinadas creencias y rituales, que se da al recién nacido sirve de base para comprender algunos de los rasgos fundamentales que constituyen las relaciones familiares y los lazos de parentesco de los akamba que habitan en el área rural de Mwuala, en el condado de Machakos, considerado desde los etnógrafos tradicionales como la tierra de los $k a m b a$, y que éstos, desde una construcción de la identidad étnica no exenta de polémicas y ciertos atropellos, han atribuido como el territorio akamba. La etnicidad, como sabemos, presenta un "carácter problemático" ${ }^{4}$, que se subraya en la cualidad de ser "polisémico" y con las "limitaciones de las definiciones extremas primordialistas" que inciden en "la necesidad de revisar el sentido de lo étnico" 5.

Describir puede llevar, desde la fascinación que produce el encuentro con el otro, con lo que se percibe como diferente, a esas "identidades ajenas" ${ }^{6}$ como identidades que "se fijan, se generalizan y se vacían de especificidad". Es por ello, que a lo largo del periodo de convivencia e interacción, se recogieron datos relevantes a cerca de las relaciones familiares, sociales y las formas de relación de esta comunidad rural, que se autorreconoce y autoidentifica como parte de los kamba de Machakos, y que por ende, identifica estos aspectos como parte de su identidad cultural y su patrimonio intangible más preciado y casi intocable.

Tras la recogida de datos, su transcripción y lectura, se procedió a una redacción sobre los aspectos fundamentales relacionados con las estructuras familiares

2 Á. Díaz de Rada, Los primeros de la clase y los últimos románticos. Madrid: Siglo XXI, 1996, p. 36.

3 R. S. Molina; P. Tomé y M. Á. Valencia, "Nuevos tiempos, nuevas familias: Aproximaciones etnográficas en el estudio de configuraciones familiares contemporáneas", en Revista Latinoamericana De Estudios De La Familia, vol. 1 (2009), p. 25.

4 M. L. Pérez Ruiz, "El problemático carácter de lo étnico", en CUHSO - Cultura-HombreSociedad, vol. 13, n. ${ }^{\circ} 1$ (2007), pp. 35.

5 Pérez Ruiz, "El problemático carácter de lo étnico", p. 36.

6 S. B. Devalle Bustamante, "Etnicidad e identidad", en Estudios de Asia y África, vol. XXXIV, n. ${ }^{\circ} 1$ (1999), p. 49. 
y sociales, los modos de relación y, centrándonos en los nombres dados al nacer a cada uno de los miembros de la familia y que tienen un significado especial entre sus componentes, y en el contexto del clan. Partimos, como consecuencia, no tanto del gran interrogante que planteábamos, sobre quiénes son los kambas, qué es ser kamba; nos centramos más bien en la hipótesis de que si conocemos y comprendemos los nombres tradicionales que utilizan entre si los kamba de esta región, llegaremos a conocer algunos elementos que constituyen sus modos de relación y costumbres de la actualidad.

Con respecto a los objetivos que pretendemos, podemos destacar los siguientes:

1. Realizar una descripción, a partir de las notas tomadas desde la observación participante, de los modos de relación de los kamba de la zona rural en la que el trabajo de campo ha tenido lugar.

2. Comprender los modos de relación que conllevan los nombres dados al nacer a cada uno de los miembros de la familia.

3. Observar el dinamismo y la incorporación de elementos que se consideran como ajenos o diferentes a lo que se percibe como tradicional. Este aspecto, se subraya como especialmente relevante entre los miembros, y con relación a los componentes de la familia que han pasado o pasan parte de su vida fuera del contexto en el que nos situamos y desde el uso de otras lenguas ${ }^{7}$.

Dar a conocer los aspectos significativos relacionados con el patrimonio inmaterial de los kamba. En este sentido, siguiendo a M. D. de Figueredo, nos centramos en aspectos que se relacionan "the notion of cultural assets" que " expresses the modern anthropological conception of culture, according to which "the emphasis is on social relationships, or even in symbolic relations" (p. 1039). Como consecuencia, nos centramos en analizar modos de relación y elementos significativos ante los que el individuo responde y la comunidad sitúa sus expectativas.

En lo referente a la metodología y muestras, se debe destacar los aspectos que siguen a continuación:

a) Se llevaron a término entrevistas a diversos miembros de la familia con la que se convivió durante este periodo. Éstas recayeron sobre las personas de mayor edad (mwuaitu), principalmente, por conocer con mayor profundi-

7 Este hecho, en especial, se ha percibido en el caso de los familiares que trabajan en grandes ciudades, principalmente, Nairobi y Mombasa, en las que sus lenguas vehiculares son el Kiswajili o el inglés.

8 M., Dantas de Figueiredo "The effects of safeguarding on ways to organize, produce and reproduce intangible cultural heritage", en Pasos. Revista de Turismo y Patrimonio Cultural, vol.13, no5 (2015), pp.1-15 
dad el conjunto de tradiciones, y por la autoridad que tiene al frente de la familia (nuclear y extensa).

b) Las entrevistas, de igual modo, también se hicieron extensivas al resto de miembros, pertenecientes a distintas generaciones.

c) Se utilizó la observación participante como medio de conocer los aspectos más relevantes de las costumbres y tradiciones familiares.

d) Con respecto a la muestra, se ha de destacar que se tomaron como referencia los 23 miembros de la familia que habitan en el hogar. Pero, de igual forma, se interaccionó constantemente con otras 14 familias de la zona rural, y que conforman una pequeńa comunidad, dependiente de Mbuini, en el condado de Machakos.

e) Las entrevistas se realizaron, utilizando para ello la lengua inglesa. Por ello, y debido a que no es la lengua vehicular principal de los kamba, se encontraron determinadas dificultades, derivadas de las múltiples traducciones. Durante el tiempo de convivencia, se llegó a conocer algún léxico y estructuras gramaticales de la lengua kikamba, como complemento a la observación.

Con el objeto de llegar a la comunidad y ampliar el conocimiento de ésta, se procedió a la técnica de muestreo bola de nieve?. Mediante ésta la familia nuclear con la que se convivió, se abrió la perspectiva al conocimiento de otras familias y miembros de clanes de Mwala, Mbui, MboonaHills, Machakos y Makutano. La característica común es que todos los sujetos pertenecían al ámbito rural, o al menos, procedían de éste y tenían arraigo familiar en las zonas interiores del condado.

\section{Contextualización. Kambas en el contexto de Kenia}

Es difícil subsumir la diversidad de un pueblo como los kamba a la reducción de unos principios derivados de la observación. Honorio Velasco y Díaz de Rada subrayan que "el holismo como operación de conocimiento practicada por el antropólogo y, en su caso, por el etnógrafo" implican que "el todo, la totalidad y el holismo son categorías a definir a nivel de la teoría y el método, no en el nivel del objeto entendido como realidad pre-teórica". Y dado que "las construcciones del todo dependen de los motivos de los investigadores" y que "no hay un único todo" ${ }^{10}$. En este caso, de las vivencias y convivencia con mwuaitu y el resto de la familia, conocemos aspectos fundamentales de la cultura kamba: lengua, costumbres, tradiciones y creencias en general, que han sido transmitidas por mwaitu a

9 M. á. Cea D’Ancona, Metodología Cuantitativa: Estrategias y técnicas de investigación social. Madrid: Síntesis Sociológica, 2001.

10 Á. Díaz de Rada, "Las formas del holismo. La construcción teórica de la totalidad en etnografía”, en Revista de dialectología y tradiciones populares, vol. 58, n. ${ }^{\circ} 1$ (2003), pp. 238. 
sus hijos, como seña del patrimonio intangible más sagrado. Sagrado, en sentido más religioso y más profano, al mismo tiempo. A fin de cuentas, como indica Olivera, primero nace la hierofanía (manifestación de lo sagrado), seguida del mito (transmisión), luego del rito (teatralización)" ${ }^{11}$. En este caso, y en tanto que se manifiesta como conjunto de aspectos esenciales para la vida de la comunidad cultural ante la que nos hallamos, los elementos vividos, interiorizados, naturalizados, no son sino una verdadera manifestación de los percibido como sagrado, en el sentido de no profanable, digno de veneración y respeto absoluto. Estos contenidos se han recibido, elaborado y reelaborado, constituyendo un espacio común de prácticas, relación y comunicación. Por otro lado, en el encuentro y en la confrontación con otros, la familia, incluida mwaitu, se define e identifica, siempre sabiendo, que un contexto como el mundo contemporáneo y en el que un país diverso como Kenia no es una excepción, "esas realidades de objetos, sentidos, cuerpos e identidades" se halla "en perpetua transformación" ${ }^{2}$. No obstante, vayamos por partes. Mwuaitu significa madre, en un tono que se percibe como muy cercano y familiar, y al mismo tiempo, cargado de autoridad. Esto ya marca su relevancia entre los suyos y como representación clánica y comunitaria. $\mathrm{Al}$ acercarme a preguntarle, en diversas ocasiones, quiénes son los kamba, su respuesta, en todas las ocasiones fue andîmamusyiwakwa, mbaiwakwa ${ }^{13}$.

No obstante, conviene acercarnos a la perspectiva externa, en modo de categorización y clasificación, que se tiene sobre los kamba de la actualidad. En este sentido, no podemos perder de vista que Kenia es un país caracterizado por la diversidad étnica, que se traduce en la presencia de una multiplicidad de grupos lingüísticos, superior a los sesenta. Si atendemos a los grupos que componen el país, se ha de poner en consideración que las lenguas autóctonas, como principal aspecto de identificación dentro del colectivo étnico al que se pertenece, se clasifican en tres familias lingüísticas: Bantú, Nilotic (y para-Nilotic), y Cushitic ${ }^{14}$. Tres cuartas partes de la población, no obstante, pertenecen al grupo bantú, destacando en este sentido los colectivos étnicos kikuyumluhya, kisii y meru ${ }^{15}$. Debemos poner en relieve, en este caso, cuatro cuestiones que no pueden pasarse por alto desde esta clasificación.

11 A. Olivera, "Patrimonio inmaterial, recurso turístico y espíritu de los territorios", en Cuadernos de Turismo, vol. 27 (2011), p. 671.

12 M. Cañedo Rodríguez, "Cultura e identidad desde la óptica antropológica: una revisión teórica”, en Thémata, n. 23 (1999), p. 184.

13 "mi familia", "mi clan", "mi gente"

14 M. M. Michieka y H. K., Ondari, "A comparative analysis of the sociolinguistic profiles of English in Kenya and Uganda", en Journal of Language, Technology \& Entrepreneurship in Africa, vol. 8, n. 2 (2017), pp. 12-25.

15 J. Kigamwa, "So Many Languages to Choose from: Heritage Languages and the African Diaspora", en Handbook of Research and Practice in Heritage Language Education, vol. 1, n. ${ }^{1}$ (2017), pp. 1-10. 
1.1. Etnicidad, identificación étnica y patrimonio intangible como agentes identitarios.

Pérez Ruiz, aunque en parte ponga sobre la mesa una confrontación entre quienes quiebran el concepto de etnicidad y los que lo afirman como categoría útil, dando argumentos a favor y en contra, afirma que "lo étnico enfatiza el papel de la dominación y de la construcción social del otro como elementos esenciales", por lo que de esta manera, "justifica relaciones de dominación”, y al mismo tiempo "tipo específico de dominación que se sustenta y argumenta sobre la base de la diferencia cultural, y que se emplea para explicar y justificar relaciones asimétricas" ${ }^{16}$. En el caso que nos ocupa, diferentes grupos en la actualidad conviven bajo la identidad nacional keniana, una realidad a menudo cuestionada y cuestionable. Pero se han forjado en confrontación directa entre unos y otros, al mismo tiempo, que bajo las relaciones asimétricas entre sí, y sobre todo, bajo el influjo colonial. Por ello, el principal aspecto externo que se ha tenido en cuenta a la hora de identificar al grupo étnico ha sido precisamente, la lengua, como primer aspecto de identidad cultural y elemento del patrimonio intangible más destacado. No obstante, la categoría que se ofrece a la hora de distinguir los colectivos es la de tribu ${ }^{17}$, cuya imposición, herencia colonial e influjo británico es más que evidente. Ya Ranger se refería a esta cuestión como "the invention on tradition revisited". De ahí que afirme que "before colonialism Africa was characterised by pluralism, flexibility, multiple identity; after it African identities of 'tribe', gender and generation were all bounded by the rigidities of invented tradition"18. A pesar de la diversidad, y de superar los cuarenta distintos grupos ${ }^{19}$, hallan aspectos de convergencia, a menudo relativamente cercanos. Su dispersión a lo largo de todo el país hace evidente la distribución lingüística, la diversidad y las constantes interacciones entre los distintos grupos. A su vez, manifiesta las mutuas influencias que ejercen unos colectivos con respecto a otros y el dinamismo en la incorporación de elementos nuevos. En cualquier caso, no podemos dejar de lado que, existe una gran afinidad entre los distintos grupos que componen la actual nación keniana, en gran medida, por el origen común; y además, por las constantes influencias que ejercen unos colectivos sobre otros.

16 Pérez Ruiz, "El problemático carácter de lo étnico”, p. 35.

17 D. N. Posner, "Measuring ethnic fractionalization in Africa", en American Journal of Political Science, vol. 48, n. 4 (2004), pp. 849-863.

18 T. Ranger, "The invention of tradition revisited: the case of colonial Africa”, en T. Ranger y O. Vaughan: Legitimacy and the State in Twentieth-century Africa. Londres: Palgrave Macmillan, 1993 , pp. 62, 63.

19 Es con frecuencia dificultoso establecer un límite entre los grupos y subgrupos que componen una u otra tribu. Algunas de ellas, presentan influencias de otros colectivos, o bien, debido a las dimensiones territoriales extensas y aisladas, tienden a una cierta particularización de características que lo separan de la generalidad. 
1.2. La autoidentificación. Un aspecto curioso es el de la identificación como miembro perteneciente a una de las denominadas tribus. Además de la identidad a la que hacíamos mención, se debe poner el acento en este caso, a la autoidentificación, el sentido de pertenencia y la percepción propia de ser parte del grupo. Este hecho, por lo que se ha podido observar desde la interacción y las entrevistas del trabajo de campo, se relaciona con el encuentro con otros grupos y la apreciación de las particularidades. La identidad, por tanto, los kamba, la asumen en su encuentro y confrontación con otros grupos, de los que además, se nutren, y a lo que al mismo tiempo, trasmiten elementos culturales que parecerían o percibirían como propios:

We are not as violent. Other tribes are very violence, the struggle and struggle, they hurt, they kill. We not. We like peace. Wetalk.

We concern work (Nicholas Kiminyú, comunicación personal, 22 de agosto de 2017)

Esta referencia de uno de los miembros de la familia nos sirve de ejemplo para ilustrar varios aspectos. El primero, la idea de tribu. Un aspecto curioso observado en casi todas las ocasiones es el hecho de que los kamba, como otros grupos del país, no se refieren a sí mismos como tribu, en casi ninguna ocasión. Pero sí lo hacen para marcar las distancias con otros colectivos. Por otro lado, la construcción étnica, en este caso, relacionada con la autopercepción, se atribuye una serie de características que además, conectan con la idea general que se tiene en el país sobre este grupo. Específicamente, en lo que hemos indicado, hay una generalización fundada en diversos elementos, pero sobre todo, en prejuicios estereotipados, del apego de los kamba a la tierra y al trabajo, así como su huida del conflicto. Es por ello que Okia ${ }^{20}$ y Mwakikagile ${ }^{21}$ se cuestionen si la categorización akamba, con independencia de la cuestión lingüística, a la que no podemos restar relevancia, no estaría sino ligada a la colonia y al conjunto de relaciones que se establecieron a partir de ésta. Si se observan ciertas costumbres, de hecho, como algunas relacionadas con la comida, el consumo de té, la religión y determinadas normas, incluidos saludos y expresividad, la huella británica es más que evidente, así como el influjo no simétrico de la colonización. De este punto de vista, la cuestión de la autoidentificación en relación con la identidad y el consecuente sentido de la pertenencia adquirirían aún más consistencia.

20 O. Okia, reseña a Myles Osborne, Ethnicity and Empire in Kenya: Loyalty and Martial Race among the Kamba, 1800 to Present (Nueva York: Cambridge University Press, 2014), en African Studies Quarterly, vol. 16 n. ${ }^{\circ} 1$ (2015), pp. 127-130.

21 G. Mwakikagile, The People of Kenya and Uganda. Dar es-Salam: New Africa Press, 2014. 
1.3. La reducción a la lengua como posible único rasgo distintivo. Es obvio que la lengua ofrece un elemento de identificación y otorga sentido de pertenencia al grupo étnico en este sentido. Los $k a m b a^{22}$, debemos destacar que éstos suponen el $11 \%$ de la población del país, lo que se correspondería a un número de hablantes que alcanza los cuatro millones, y que se halla en proceso de expan$\operatorname{sión}^{23}$. La mayoría de los kamba se sitúan en el actual condado de Machakos y otros limítrofes en Kenia. Si bien, y a pesar de las demarcaciones, se extienden por otras regiones, tanto del país como de Tanzania y Uganda (Mwakikagile, 2014). De igual modo, y aunque pueda resultar un dato insólito, existe una comunidad kamba en Paraguay, los kambacuá, descendientes de un grupo de esclavos liberados y que están constituidos en la actualidad por más de diez mil representantes, muy conocidos por conservar música y danzas tradicionales ${ }^{24}$. En cualquier caso, responderíamos a un sistema excesivamente simplista y reduccionista si tratáramos de referirnos a los kamba, como grupo cultural basándonos sólo en la lengua.

1.4. La construcción histórica de la búsqueda identitaria en clave localista ${ }^{25}$. Habitualmente, se identifica Machakos con la región propiamente kamba, aunque sólo la mitad de representantes de este grupo étnico habita en este condado. Este hecho es debido a que esta región semiárida cercana a Nairobi se muestra como la más homogénea en cuanto a la lengua kikamba se refiere, tanto en su uso cotidiano, como en la uniformidad frente a otras variantes. No obstante, se ha de indicar que forma parte de los kamba, precisamente, su constante proceso de interacción con otros grupos, el dinamismo de su "cultura", y la facilidad de incorporación de elementos, aparentemente, procedentes del exterior, de lo percibido como alteridad. De todos modos, y siguiendo a Díaz de Rada (2008), "poco podemos aportar a la comprensión de un campo de relaciones étnicas si lo contemplamos de una vez por toda a la luz de los esquemas categoriales de la burocracia política (p. 212). No se puede dejar de lado que Machakos no es sino un condado, una división administrativo-política, que hunde sus raíces en divisiones territoriales funcionales que parten de la época colonial. Por otro lado, se ha de

22 A menudo, también se emplean, según se ha derivado de la observación sistemática, los términos wakamba, para el plural, akamba, en el caso de los individuos representantes del grupo étnico.

23 M. Mwaniki, "Language management and devolved governance in Kenya”, en South African Journal of African Languages, vol. 37, n. 2 (2017), pp. 211-223.

24 J. Lipski, "Afro-Paraguayan Spanish: The negation of non-existence", en Africology: The Journal of Pan-African Studies, vol. 2, no. 7 (2008), pp. 2-32. L. Á. López, "200 años de herencia lingüística afrolatina: descendientes de Ansina y otros soldados de Artigas en el Paraguay”, en $M o$ derna språk, vol. 107, n.o 1 (2013), pp. 1-10.

$25 \mathrm{Me}$ refiero en este caso a la identificación con un lugar como patria de esa cultura imaginada y/o construida a raíz de una serie de hechos históricos, migraciones y demás eventos de fondo histórico y, a menudo, legendario. 
señalar que, si tenemos como punto de partida y referencia la lengua, considerada en este caso como elementos de identificación ${ }^{26}$, se ha de poner en relieve cómo el kikamba comparte alrededor del $60 \%$ de su léxico y gramática con otras lenguas bantúes cercanas y contiguas, tales como el Gikuyu, Embu, Chuka y Meru ${ }^{27}$ (Roberts-Kohno, 2000).

Tabla 1. Distintas variedades del kamba

\begin{tabular}{ll}
\hline Ulu+ & Mumoni* $^{*}$ \\
\hline Nganyawa+ & Kitui* $^{*}$ \\
\hline Kinabgo* & Kibwezi* $^{*}$ \\
\hline Kisuari* & \\
\hline
\end{tabular}

+ Se corresponde a las dos variedades generales de la lengua kamba. * Son variantes relacionadas con los lugares

Fuente: elaboración propia, basada en Hobley (1971) y Hinde (2014)

De cualquier modo, como subrayan Mercado Maldonado y Hernández Molina ${ }^{28}$, en los contextos en los que los grupos humanos fluyen, como ocurre en la actualidad en casi todos los espacios, y del cual, este condado y los aledaños no suponen una excepción, "la construcción del sentido de pertenencia se dificulta, ya que los sujetos están adscritos a diversos grupos", por los que "los sujetos, a través de sus múltiples interacciones cotidianas van seleccionando los repertorios culturales que responden a sus intereses y aspiraciones personales, así como a las exigencias sociales" (p. 229). En realidad, por el dinamismo que se manifiesta del proceso que se atribuye a los kamba, en su historia y asentamiento, esta realidad ha sido una constante. $\mathrm{Y}$ en lo que concierne a la actualidad, se ha de entender que hay una definición e identificación en la relación con la alteridad. Es ahí donde se buscan los principios más arraigados de la cultura, presupuesta e imaginada, pero vívida y dinámica al mismo tiempo. "Asyawakikamba” es una de las senten-

26 Hemos de recordar cómo Molano y Lucía (2007) indican que la "lengua es instrumento de comunicación entre los miembros de una comunidad, las relaciones sociales, ritos y ceremonias propias, o los comportamientos colectivos" (L., Molano, \& O., Lucía, "Identidad cultural un concepto que evoluciona”. En Revista Opera, vol. 7(2007), pp. 1-12. p. 73). De ahí que se contemple desde esta perspectiva como un elemento de identificación que otorga sentido de pertenencia y homogeneización, en una realidad dinámica y cambiante como es la "cultura".

27 A. N. Kioko, "The Kikamba Multiple Applicative: a problem for the lexical functional grammar analysis", en South African Journal of African Languages, vol. 15, n. 4 (1995), pp. 210216. Cfr. R. R., Roberts-Kohno, Kikamba phonology and morphology. Tesis Doctoral, The Ohio State University, 2000.

28 A. Mercado Maldonado y A. V., Hernández Oliva, "El proceso de construcción de la identidad colectiva”, en Convergencia, vol. 17, n. 53 (2010), pp. 229-251. 
cias más repetidas por las mujeres de mayor edad de la familia y la comunidad a los miembros más jóvenes:

Mwuaitu is mad at us when we speak Kiswajili. She understands. But she concerns we ought to speak kikamba. We are at home, in our land. This annoys our ancestors. And mum, buried over there. She's funny. We always speak Kiswuajili in Nairobi. My friends are Giguyu and Luyha. They don't know Kikamba. I forgotlotsofwords (Sammy Kimeu, Comunicación personal, 28 julio de 2017)

Los kamba dominan más lenguas, en especial, entre los sectores más jóvenes y que han tenido la oportunidad de estar escolarizados durante periodos más prolongados. Kiswajili e inglés son utilizadas por los kamba, por ser lenguas oficiales en Kenia. No obstante, se observa que, a nivel familiar, el uso de ambas es casi inexistente. Sólo se utiliza el kamba. El kiswuajili, por su parte, y debido probablemente a los cambios en las distintas políticas lingüísticas, presenta un mayor nivel de competencia entre los jóvenes. Se usa, no obstante, como instrumento para la comunicación interétnica, con otros colectivos del país. Por su parte, tanto inglés como kiswajili se hallan condicionados por el nivel educativo de la persona. Hay diferencias significativas, marcadas por los sectores.

Si tratamos de profundizar en el origen de los kambas de la actualidad, hemos de poner en relieve que resulta dificultoso indagar en sus raíces, siguiendo pesquisas es investigaciones constrastivas. Se debe indicar, siguiendo a Pina ${ }^{29}$, en los contextos multiculturales, fenómeno, por otro lado, nada nuevo, la búsqueda y la construcción de principios y arraigos definitorios se torna en un afán constante.

Se considera que, en origen, el grupo emigró desde la zona oeste de la actual Tanzania hacia las inmediaciones de la meseta que ocupa los márgenes del este de Nairobi, en la región ocupada por el pueblo Nyamwezi. Éste, uno de los mayoritarios en Tanzania, habría sido desplazado parcialmente por los kambas, o se habría procedido a una sucesión de interacciones que habrían dado espacio a asimilaciones, convivencia y surgimiento de un colectivo ${ }^{30}$. Una de las teorías más extendidas en la actualidad es la que subraya que los kambas son producto del cruce interétnico de diversos grupos que se cruzaron por distintas alianzas matrimoniales, y por ende, forjaron sus señas de identidad a partir de este conjunto de interrelaciones (Mwakikagile, 2014). durante la presencia británica, los kamba

29 M. B. Pina, La construcción de la identidad en contextos multiculturales (vol. 149). Madrid: Ministerio de Educación, 2000.

30 R. G. Abrahams, The Peoples of Greater Unyamwezi, Tanzania (nyamwezi, Sukuma, Sumbwa, Kimbu, Konongo). East Central Africa. Oxon/Nueva York: Routledge, 2017. S. J. Rockel, "'A nation of porters': The Nyamwezi and the labour market in nineteenth-century Tanzania”, en The Journal of African History, vol. 41, n. 2 (2000), pp. 73-195. 
establecieron relativas buenas relaciones, y tomaron parte del ejército y las fuerzas públicas. En este sentido, se ganaron la fama entre los colonizadores de estructurados, dóciles y trabajadores, por los que éstos no procedieron, presuntamente, a una imposición muy brusca de sus estructuras sociales. De ahí, lo que expresábamos previamente, relativo a la influencia colonial en los ejercicios de delimitación y definición de identidades, a menudo muy problemáticas y artificiales.

Si tenemos en cuenta la tradición oral de este pueblo, que ha permitido su arraigo patrimonial inmaterial, y por ende, su sentido de pertenencia, y en referencia a sus orígenes, podremos poner en relieve que aún se hallan historias que sitúan el origen de los kambas en los alrededores del monte Kilimanjaro ${ }^{31}$, desde donde emigraron hasta situarse en Mbooni y algunas de las zonas en las que actualmente se sitúan. De ahí, que aún en el colectivo, se hallen presentes mitos y creencias del pasado, que son difíciles de cifrar en cuanto a su origen, y que se han transmitido de forma oralmente de forma intergeneracional. Entre estos aspectos se halla la creencia en el Ngai o ggaiMumbi $^{32}$, máxima deidad, compartida con otros pueblos cercanos como los kikuyu.

Tabla 2. Principales localidades con población Akamba

\begin{tabular}{lll}
\hline Condado de Machakos & Condados limítrofes & Otros lugares \\
\hline Machakos & Kiivaani & Nairobi \\
Mwuala & Kayunvu & Mombasa \\
Makutano & Mbooni & Kajaido \\
Mtungulu & Muranga & Taveta \\
AtiRiver & & Ukunda \\
Masii & & \\
Mbuini & & \\
Syathani & & \\
Pakhaline & &
\end{tabular}

Fuente: elaboración propia basada en visita, observación y fuentes de información municipales.

31 Esta perspectiva fue defendida por antropólogos desde hace un siglo, especialmente por Middelton y Lindblom, primeros en referir el pueblo kamba (J. Bale y J. Sang, Kenyan running: movement culture, geography and global change. Oxon/Nueva York, ECA/Routledge, 2013; J. Mbiti, reseña a K. Ndeti, Elements of Akamba Life [Nairobi: East African Publishing House 1972], en Afri$c a$, vol. 44, n. 4 [1974], pp. 429-429). Estas teorías, hoy día, se hallan muy matizadas por diversos estudios y responden a una perspectiva etnográfica en exceso esencialista.

32 G. N. Wamue-Ngare y W. N. Njoroge, "Gender paradigm shift within the family structure in Kiambu, Kenya”, en African Journal of Social Sciences, vol. 3 (2011). pp. 10-20. 
Al ser Machakos una zona de dominio lingüístico kikamba, ha sido considerada como la tierra de los akamba. En cualquier caso, y derivado de la observación, se puede decir que, a las variantes de esta lengua bantú se han de añadir las locales. De la misma forma, la diversidad alcanza a la distinción entre espacios urbanos y rurales. En ocasiones, en este sentido, se perciben divergencias. Si bien, hay una nivel de comunicación fluida entre los hablantes akamba de zonas muy alejadas. Pero, de igual forma, se da una comunicación fluida entre éstos y los pertenecientes a otras tribus ${ }^{33}$, y manteniéndose en ambos casos, las lenguas originarias vernáculas. Esto manifiesta cercanía lingüística e influencias constantes. Como subraya Díaz de Rada (2008), "la relación interétnica no divide dos zonas homogéneas, demarcables e identificables con dos sujetos sociales alternos" (p. 2005). La frontera cultural, marcada en clave de profunda artificialidad quedaría quebrada en este caso ${ }^{34}$. Giménez (2009), aunque subraya de manera crítica que "la fuerza de una frontera étnica puede permanecer constante", no se puede pasar por alto que "en la interacción con los otros grupos se define la identidad" (p. 7).

\section{NaCimiento a ASIGNACIÓN DE NOMBRE COMO ELEMENTO DE INTEGRACIÓN,} PERTENENCIA, RELACIÓN E IDENTIDAD

Fertilidad y nacimiento se consideran como aspectos básicos en la familia. Se sitúa el sentido y la teleología misma de la existencia en la procreación. De ahí que la llegada de un nuevo ser se viva como todo un acontecimiento, y la fertilidad, como la mayor de las bendiciones que pueda otorgar Mulungu, Ngai $i^{35}$ (Langmia, 2016), esto es, la divinidad, o la misma naturaleza, a la que el akamba se siente completamente ligado por pertenencia y sentido de unidad. El hecho de tener descendencia se torna en un requisito casi indispensable para este colectivo ${ }^{36}$. No se puede dejar de lado que la ausencia de hijos se comprende como una especie de maldición, porque no otorga sentido completo de plenitud a la familia. Este

33 Se utiliza este concepto, como antes se indicó, para mantener la terminología que se aplica en el país y sin renuncia a la crítica sobre su contenido.

34 G. Giménez, "Cultura, identidad y memoria: Materiales para una sociología de los procesos culturales en las franjas fronterizas”, en Frontera norte, vol. 21, n. 41 (2009), pp. 7-32.

35 Como indica Gitari M. D. Gitari (Concepts of God in the traditional faith of the Meru people of Kenya. Tesis Doctoral, University of South Africa, 2009), esta denominación de la divinidad es propia de los kikuyu, aunque también se utiliza tradicionalmente por parte de los akambas. Este hecho muestra el dinamismo cultural, la interinfluencia y, al igual modo, el origen compartido de los pueblos. Cfr. K. Langmia, "Traditional African and Western Modern Cultures”, en K. Langmia: Globalization and Cyberculture. Londres: Palgrave Macmillan, 2016, pp. 5-20.

36 Durante el periodo de convivencia, se constató la presencia de varias religiosas en la familia. Por su estilo de vida, eran un referente en la comunidad. Pero siempre se las refería desde una cierta incomprensión, por no cumplir con su función presupuesta más importante, según ellos, a nivel familiar: tener descendencia. Al preguntar sobre la cuestión, afirmaban, los hombres mayores de la familia, que cuando muera, ya no existirán, no habrán dejado semilla en esta tierra, y serán sólo del mundo de espíritus. 
hecho, por los paralelismos que se establecen, es aún más dramático en el caso de la mujer, que se compara con la tierra. En cualquier caso, este hecho, tal y como hemos indicado con anterioridad, se relaciona con los roles de género que se atribuyen en el seno de las comunidades kamba.

Hemos de tener en consideración que el nombre dado al nacer otorga a la persona una característica y una idiosincrasia particular, que le confiere identidad, un modo de relación e incluso una forma de ser. La mayoría de los akamba, profesan el cristianismo, en su profesión protestante o católica, y por ello, tienen un primer nombre de bautismo, generalmente, cristiano. No obstante, su segundo nombre es kamba. El nombre se convierte en el elemento de identificación dentro de la familia, el clan y la comunidad. Parte, esta comunidad de origen bantú, de considerar que la manera de denominar al individuo es una característica que se conforma en un rasgo de su personalidad. En cualquier caso, se ha de destacar que ésta, aunque con matices, parece ser una característica, por lo que se ha observado, que no sólo se extiende entre los kambas de las zonas rurales, sino que además, es propia de otros grupos del país. Lo que sí se muestra como más evidente, es que, a mayor urbanización, esta costumbre parece diluirse más. Se optaría por el nombre oficial asignado.

Se debe tener en cuenta, además, que el nombre elegido se asocia a algún acontecimiento que tiene que ver con la concepción, el periodo de gestación, y sobre todo, el momento del nacimiento. De esta manera, el nombre tradicional se hallará ligado a los eventos de la cotidianidad, y se convertirá en un rasgo constante a lo largo de la vida de la persona. En cualquier caso, y derivado de la observación directa, se puede destacar que hay una utilización selectiva del nombre, dependiendo del contexto. Así, a nivel familiar (tanto en la familia nucleas como en le extensa), se conoce a la persona y la denomina según su nombre tradicional kamba. Por el contrario, en el contexto de relación con otros grupos étnicos, extranjeros o en núcleos de población mayores, como la misma ciudad capital, se recurre a la identificación por medio del nombre cristiano dado en el bautismo. Además de este hecho, se ha de tener en cuenta, de que a los dos nombres, se añade el de la familia, a modo de apellido. Esta circunstancia difiere de otros colectivos presentes en Kenia. Además de lo que hemos descrito, se ha de destacar que, entre grupos étnicos cercanos y en constante interacción, en ocasiones sí se utiliza el apelativo tradicional en kikamba, bien porque su significado se comprende con facilidad (por afinidad lingüística), o bien por reivindicación étnica y como modo de reafirmar la identidad familiar, y por ende, clánica.

En lo referente a la naturaleza de los nombres tradicionales, desde las entrevistas y observación realizada, pudimos extraer diversos campos léxicos sobre los que se eligen los nombres, y que sintetizamos a continuación.

2.1. Contexto en el momento del nacimiento. En este caso, se distinguía con claridad algún acontecimiento relativo al parto. 
a. Momento del día (luz, noche, mañana)

b. Fenómenos de la naturaleza (lluvia, niebla, sol).

c. Circunstancia o momento concreto del nacimiento (demora en la concepción, periodo de embaraza más prolongado de lo que se esperaba, parto complejo y dilatado, etc.).

2.2. Lugar que ocupa en la familia

a. Primogénito, que en ocasiones, lleva el nombre familiar, generalmente, el del abuelo.

b. Benjamín de la familia.

c. Nacido tras un hermano inmediatamente mayor fallecido, o tras un aborto. En este caso, se celebra especialmente el nacimiento como una bendición tras el dolor de la pérdida previa. De ahí que el nombre katunge se perciba como el perteneciente a una persona especial, porque equivaldría a alguien que viene a reemplazar a quien ya no está.

Katunge means who is born back into the family again. In my case, there was a baby called Veronica Kiasyo. She was born before me. She had fever and malaria and died I days, I guess. You can imagine my family's pain and suffering. Then, as other girl was born, in this case me, following our traditions, she must be called Katunge or Mutunge. It means that the girls who died, Veronica, in the case of my family, is born again in the family. It's reason for joy. It's like if she takes away sadness, and the family comes back to happiness (Magdalena KatungeKiminyú, comunicación personal, 12 septiembre de 2017).

2.3. Nombres relacionados con características familiares o personales de los progenitores. En ocasiones, en estos casos, se pueden percibir con un cierto tono despectivo o en clave de compasión ${ }^{37}$.

2.4. Animales. Éstos se usan como característica del recién nacido, percibida antes del parto, o para alejar maldición. De igual manera, ante niños que han nacido muertos, o que aparentemente lo estaban. De esta manera, se aleja el mal espíritu. Además de esto, es una forma de reservar los nombres percibidos como mejores para los posteriores hijos. En la interacción con la familia y el clan, todos habían oído hablar de este fenómeno, y así lo reprodujeron. Pero no conocían a nadie en la actualidad que tuviese ese nombre.

37 Valga el ejemplo de munyoki, que hace referencia a la afición al alcohol del progenitor. 
NgukuMbuku. No. I Heard once in my clan. But that's strong. To call a son $N g u k u$. Even worst Mbuku. You won't find respect. That's not actually such a common issue. You must think twice $(\ldots)^{38}$ do (Mwuaitu, comunicación personal, julio de 2017)

2.5. Expectativas puestas sobre el recién nacido. En este caso, se proyecta sobre el neonato el deseo, anhelo o intención de los padres. Al igual que sucede con la anterior categoría, estos nombre son mucho menos frecuentes, aunque también se hallan vigentes.

Tabla 3. Nombres comunes dados a los recién nacidos

\begin{tabular}{|c|c|c|}
\hline Mutuku & Katuku & Nacido/nacida durante la noche \\
\hline Kioko & Kiloko & Nacido/nacida durante la mańana \\
\hline Mutuo & Mutua & Se hizo esperar para nacer \\
\hline \multicolumn{3}{|l|}{ Makunbi* } \\
\hline & Katee* & \\
\hline & Muthike & \\
\hline Muthinda & Muthindi/Indinga & Tardó en nacer \\
\hline \multicolumn{3}{|l|}{ Muli } \\
\hline \multirow[t]{2}{*}{ Kyalo* } & & Nació durante el viaje \\
\hline & Mueni & Nació en presencia de visita \\
\hline Wendo & Mwende & Quien es amado/a quien se da amor \\
\hline Mbua/Kimeu & Mumbua & Nació durante el tiempo de lluvia \\
\hline $\begin{array}{l}\text { Katunge/Ndunge/ } \\
\text { Mutunge }\end{array}$ & $\begin{array}{l}\text { Kasyoka/Musyoka/ } \\
\text { Mutunga }\end{array}$ & $\begin{array}{l}\text { Vino a reemplazar al que murió y a } \\
\text { restaurar la alegría }\end{array}$ \\
\hline Mutinda/Mwikali & & Tardó en nacer \\
\hline
\end{tabular}

Fuente: elaboración propia basada en la observación participante.

*Se corresponden con nombres que para los que no se ha encontrado femenino, o que no tienen traducción al inglés.

De la observación y las conversaciones obtenidas, se deriva que el nombre asignado se interpreta como una característica que va a acompañar a la persona durante toda su vida, condicionando su destino, influencia en la familia y la comunidad, y a su vez, forma de ser. De hecho, algunas ancianas comentaban que, antes de na-

38 Se indica en este caso con el presente signo la imposibilidad de transcribir varias palabras de la conversación. 
cer, el bebé le sugería a la madre su nombre. Y si ésta no lo escuchaba, se hacía notar esta voluntad por medio de hechos y acontecimientos de mayor envergadura.

A continuación, presentamos algunos otros nombres que aparecen con menor frecuencia, y que refieren a las cuestiones que hemos indicado con anterioridad.

Tabla 4. Nombres relacionados con animales o con expectativas que se sitúan en el recién nacido

\begin{tabular}{ll}
\hline Munyambu & León \\
Nguku & Pollo \\
Mbuku & Liebre \\
Ngiti & Perro \\
Mbiwa & Zorro \\
Kitonga & Será fuerte \\
Mutongoi & Para ser líder \\
Muthui & Rico \\
Mumbe & Hermosa \\
\hline
\end{tabular}

Fuente: elaboración propia

Como se indicó previamente, estas formas de denominar a los menores no se encuentran con frecuencia. Tan sólo se ha podido conocer a un varón llamado Muthui. De igual modo, ninguna persona de las entrevistadas o con las que se tuvo conversaciones conocía a nadie que llevara un nombre particular: Msumbi (rey). Pero es un nombre, según se deriva de lo compartido, que se reserva para personas con un liderazgo que vayan a llevar a cabo acciones determinadas, encaminadas a la liberación o la transformación colectiva del pueblo.

\section{Nombre, MOdo DE RELACión y ESTRUCTURA FAMiliar}

Lo que no se nombra no existe (George Steiner)

Como se ha indicado y descrito, en el seno familiar, la forma de nombrar a una persona desde su nacimiento condiciona en parte los modos de relación y supone toda una seña de identidad. Aunque se podría pensar, siguiendo las pautas de numerosos autores, que manifiestan la disolución del sujeto en el grupo debido a "la variedad de experiencias", no obstante, existen una serie de elementos que 
impiden la disolución absoluta de la identidad y que anclan al sujeto a una determinada identidad personal, aunque de forma problemática, conflictiva, matizada y cambiante" (Revilla, 2003, p. 54). No podemos dejar de lado la problematicidad en sí del concepto y del contenido identitario, tanto a nivel individual como en el grupo. Y de las polémicas y conflictividades que podemos encontrar en el proceso de cierne de la identidad, tanto personal como grupal. No se puede olvidar la profunda relevancia que posee el grupo, y en este caso, la familia (cercana y amplia), y además, que "el enredo de las identificaciones se procesa en la escala de la vida concreta, práctica, local” (Díaz de Rada, 2008, p. 208).

Desde la manera de dirigirse y denominarse, así como desde el significado de ésta, podríamos afirmar, aún cayendo en el peligro de tender "siempre a percibir y definir sujetos compactos, claramente definidos, y por tanto, demasiado groseros para el examen etnográfico de los agentes y sus prácticas" (Díaz de Rada, 2008, p. 216), que la familia nuclear parece constituir el elemento fundamental desde el que parten el resto de redes de relación. En cualquier caso, y de igual modo, la familia extensa también se torna en una red de relevancia, al igual que el clan al que se pertenece. Los lazos de solidaridad y la unión de sus miembros pueden llegar a ser, con frecuencia, muy intensos. Y por otro lado, se establecen modos de relación, roles y muy demarcados en el seno de las estructuras de parentesco de los kamba ${ }^{39}$ (Díaz Delgado Raitala, 2016).

Como parece lógico, el nombre kamba es el elemento de nexo y unión entre los miembros de la familia a muchos niveles. Pero de manera igualmente evidente, hay una forma de dirigirse al familiar, según el pariente. Este hecho se hace extensivo no sólo a la familia, sino también al clan ${ }^{40}$.

Se ha de indicar que, derivado de la observación participante, se puede subrayar la relevancia de la familia entre los kambas, constituyendo el núcleo de estructuración social central del que parten todos los demás sistemas de relación, aunque, como describiremos posteriormente, el sentido de pertenencia clánico y comunitario otorgaarraigo.

39 D. Díaz Delgado Raitala, Bridewealth: an ethnographic study on the narratives and descriptions of the practice of bridewealth establishing its purposes, effects and consequences among the Luo, Kikuyu, Kalenjin, Meru, Kamba, Maasai, Kisii and Luhya ethnic groups in Kenya. Tesis Doctoral, University of Jyväskylä, 2015.

40 En distintos encuentros y visitas de parientes lejanos o miembros del clan, se me presentaron como tios, madre, padre, hermano, respecto a la familia o al sujeto con el que estaba en ese momento, denominándolos así por correspondencia clánica 
Tabla 5. Estructura familiar y clánica.

\begin{tabular}{ll} 
& Términos básicos \\
\hline Mbai & Clan \\
Familia & Musyi \\
Tata & Padre-cabeza de familia \\
Mwaitu & Madre \\
Usua & Abuela \\
Umaa & Abuelo \\
Mwendw'au & Tío paterno \\
Inaimiwa & Tío materno \\
Mwendya & Tía materna \\
Wa-asa & Primos paternos \\
Wamwendya & Primos maternos \\
\hline
\end{tabular}

Fuente: elaboración propia

La tradición marca que la familia kamba siga un sistema de patriarcado. La transmisión del apellido, las posesiones y las decisiones se dan por línea paterna. De igual modo, existe una escisión de roles que separa género en cuanto a los papeles que cada uno juega en las labores familiares y modos de relación social. En cualquier caso, y a pesar del arraigo a determinadas creencias y comportamientos, se puede indicar que la familia kamba se halla en pleno proceso de cambio, de transformación, por lo que hay una brecha generacional fácilmente perceptible entre las nuevas generaciones y las más veteranas. Como Moghadam señala, "modern family has two main functions: to socialize children into society's normative system of values and inculcate appropriate status expectations, and to provide a stable emotional environment" ${ }^{41}$. Como afirman Wamue-Ngare y Njoroge, no proyectan sino un auténtico "genderParadigm Shift withinthefamilystructure" 42. A pesar de estos procesos de transformación, y de la perspectiva nueva que está tomando la familia en este contexto, que en ocasiones toman un cariz comunitario $^{43}$, los roles que podrían considerarse como tradicionales aún se conservan en clave de este patriarcado. Este hecho se refleja en varios aspectos que se han podido derivar de la observación participante:

41 V. M. Moghadam, "Patriarchy in transition: Women and the changing family in the Middle East", en Journal of Comparative Family Studies, vol. 35, n. 2 (2004), p. 137.

42 Wamue-Ngare Njoroge, "Gender paradigm shift...", p. 10.

43 J. Muthuki, "Challenging patriarchal structures: Wangari Maathai and thegreenbeltmovement in Kenya”, en Agenda, vol. 20, n. ${ }^{\circ} 69$ (2006), pp. 83-91. 
1. La economía del hogar. Aunque la administración corre cargo de la mujer, es el tata quien proporciona la mayor parte de los ingresos. De igual manera, se considera que éste es quien realmente sostiene el hogar, aun en casos en los que la mujer es la que tiene un empleo y proporciona la entrada de ingresos ${ }^{44}$.

2. La toma de decisiones importantes. Cualquiera de éstas suele estar a cargo del padre de familia. Si bien existe un diálogo en torno a éstas, también es cierto que se comunican por vía paterna. En las decisiones relativas a los hijos, éstas descansan sobre el patriarca, aunque la labor de mwuaitu, esto eso, la madre, no sólo es de mediación, sino de una clara influencia condicionante sobre el esposo.

3. Propiedad y posesión. La herencia de la propiedad se da, de igual forma, por la línea paterna, aunque se ha de tener en consideración que este hecho no exime de que la mujer pueda ser propietaria de tierras o bienes. La transferencia de propiedades a los hijos puede darse por línea materna, en caso de viudedad ${ }^{45}$.

4. La costumbre del matrimonio. Primeramente, y teniendo en cuenta que no pueden emparentarse miembros del mismo clan, como describiremos con posterioridad, la pedida de mano implica la entrega de una dote al padre de la familia que vincula el precio pagado al valor que se da a la futura esposa. El acto de petición incluye un protocolo de intervenciones de cada uno de los participantes, la declaración de intenciones y el pago de la dote. Aunque los padres realizan diversas preguntas sobre las intencionalidades del pretendiente, es el padre quien lleva a cabo el monopolio conversacional y las cuestiones más comprometidas. De igual forma, sobre él recae la responsabilidad de dar la última palabra sobre el compromiso.

5. La instalación de la mujer tras el matrimonio, que habitualmente consiste en vivir bajo el techo del esposo y su familia. En algunas ocasiones, se puede destacar que se ha observado una situación completamente inversa, en la que es el hombre en el que se ha ido a vivir junto a la familia de la esposa. Pero esta situación está socialmente mal vista y en algunas ocasiones, se justifica por la falta de medios ${ }^{46}$.

44 A este propósito, quisiera destacar dos datos. El primero, la percepción de este hecho por parte tanto de hombres como de gran número de mujeres, muy a pesar de la cantidad de horas que ellas emplean en el día en trabajo manual, cuidado del hogar, niños y mantenimiento de la familia. Por otro lado, tuve la ocasión de convivir con dos familias cuyos cabezas familiares tenían serias discapacidades físicas que les impedían trabajar de forma remunerada. A pesar de esto, se consideraban como sostenedores de la familia, por ceder permisos a sus mujeres para trabajar.

45 En el caso específico observado, el de Rose Kiminyú, Mwuaitu, era propietaria de las tierras de su marido, y de las que había heredado de su familia. Se situaba ante la dificultad, en este caso, de proceder al reparto entre todos sus hijos, siguiendo una normativa no escrita, que ella misma encontraba difícil de interpretar.

46 El matrimonio de la tercera generación de la familia Kiminyú, formado por Nicholas y 
En lo concerniente a la función de la mujer, es necesario subrayar que ésta se muestra como el pilar de la familia, y la transmisora de la lengua, las creencias, costumbres y tradiciones. Podríamos indicar, a grandes rasgos, que aunque ambos progenitores intervienen en la adquisición de los principios básicos que rigen las reglas de relación intrafamiliar y comunitaria, es en la $M$ wuaitu ${ }^{47}$ en quien recae el peso de la responsabilidad más directa e inmediata. Entre las funciones que se atribuyen desde las expectativas familiares y grupales sobre la madre, se pueden señalar las siguientes:

1. Crianza de hijos. Transmisión de lengua, valores y principios fundamentales que se perciben como esenciales para el grupo.

2. Labores domésticas, que se relegan, en términos generales a la madre, las hijas y las nueras. La coordinación de estas actividades es llevada a cabo por la mwuaitu. En caso de convivir varias generaciones, se tiene en consideración otro criterio de relevancia: la ancianidad. Ésta otorga autoridad e infunde respecto en el resto de los miembros de la familia. En el caso concreto que analizamos, ante la viudez de Mwuaitu, ella es, por experiencia, edad y papel en la familia, la que toma las decisiones importantes, tiene la última palabra, y el verdadero espiritu de la familia. Esta percepción es bilateral, tanto por parte de ella, como del resto, quienes tienen plena conciencia de esta circunstancia.

3. Trabajo manual. No se puede olvidar que la mayoría de las familias kamba se dedican a la agricultura. Como campesinos, y en el contexto de una economía de subsistencia, Mwaitu y el resto de las mujeres, colaboran activamente en las labores del campo, esto eso, siembra, mantenimiento y recolección.

4. Oraciones y peticiones de alcance familiar. Existen rituales y celebraciones que combinan creencias del pasado con las estructuras religiosas más difundidas y presentes en la actualidad, en especial, las respectivas doctrinas católica o evangélica. Ante las distintas maldiciones, la ausencia de lluvias, la incursión en el hogar de serpientes u otros animales que pueden generar, Mwaitues la encargada habitual de las oraciones adecuadas para el momento. En la actualidad, la familia kamba se halla condicionada por la pertenencia a una parroquia o iglesia. En este sentido, se debe a sus siste-

Grace, me resultó paradigmático en este sentido. Ella se desplazó más de cien kilómetros para ir a vivir en casa de la familia de su marido tras el matrimonio. Cuando pregunté sobre la posibilidad de que Nicholas hubiera hecho el proceso inverso, todos rieron, a pesar de que en la zona de la esposa había muchos más recursos.

47 Nos referimos en este caso a Mwuaitu en mayúsculas, porque es la manera de denominarla entre todos los miembros de la familia. Pero también, por extensión, nos referimos a todas las madres que hemos conocido en Mwuala, y que por edad, revisten autoridad. 
mas de creencias o liturgias. Pero este hecho no implica la generalización de determinadas fórmulas que han perseverado a lo largo del tiempo y que, con frecuencia, han sido trasmitidas de forma oral por vía materna.

La asunción de roles de género se muestra como una evidencia dentro de los kamba, algo asumido y lógico en la transmisión de una generación a otra. No obstante, esta cuestión resulta relativa entre las nuevas generaciones, en especial, en los ámbitos más urbanos, en los que se está procediendo a un proceso de transformación en este sentido. Aunque se da una brecha significativa en el acceso a la educación y los ańos de escolarización entre hombres y mujeres, se observa un mayor interés por parte de las familias en la formación de las mujeres. No obstante, no se puede olvidar que este acceso a los centros educativos. La matriculación, materiales, distancia y dificultades de movilidad en los periodos de lluvias conllevan que si hay que optar por la escolarización, se continúe dando prioridad a los hijos sobre la educación de las hijas.

En lo referente a los clanes (mbui), hemos de indicar que se trata de estructuras amplias de parentesco entre los kamba y otros colectivos de la región. Otorgan sentido de pertenencia, de origen y de referencia ${ }^{48}$ (Middleton y Kershaw, 2017). Se han de señalar, a este propósito, varios aspectos que se han derivado de la observación en el trabajo de campo:

a. Conciencia de familia paralela. Cuando se presentan los miembros del clan respecto a los de la familia, lo hacen denominándose desde un parentesco (padre, madre, hermano, hermana, tío, sobrina, etc.). Esto se muestra desde un paralelismo que manifiesta el origen común de los miembros del clan, un tronco común de antepasados que los emparenta y que los une de forma inherente ${ }^{49}$.

b. La conciencia y reconocimiento de los miembros del clan como parte de la familia extensa conlleva una obligación inexcusable y que no puede ser relativizada bajo ningún pretexto: la prohibición de emparentarse con familiares del mismo clan.

You cannot marry to someone of your clan. It is like if you marry to a sister to you. You must marry one woman of a different clan. We are siblings, parents, daughters, sons. It is mandatory to respect that. It is an offence, and insult to the Lord (Rose Kiminyú, comunicación personal, agosto de 2017).

c. En este contexto, lo clanes han contribuido a la cohesión social y cultural. Pero, de igual modo, a la diversificación del grupo. En cualquier caso, un

48 J. Middleton y G. Kershaw, The Kikuyu and Kamba of Kenya: East Central Africa. Oxon/ Nueva York: Routledge, 2017.

49 J. A. Bailey, Echoes of Ancient African Values. Bloomington, Indiana: AuthorHouse, 2005. 
clan, para los kamba, se convierte en un punto de referencia con respecto al cual, se mantienen normas específicas y modos de relación. Del mismo modo, hay una unión basada en los antepasados que se sigue manteniendo en el presente, y que, existan o no relaciones directas, preservan una unidad, en ocasiones, virtual o utópica. El clan, de una manera u otra, constituye una base de interacciones hacia redes sociales más amplias, a pesar de las limitaciones ${ }^{50}$.

d. Entre los kamba que se han observado, el clan se entiende desde la perspectiva de una unidad más amplia y superior de parentesco ${ }^{51}$, y que se distancia del concepto tribal que se encierra "en algún conjunto de objetivaciones externas" 52 y se inserta más en una perspectiva de lazos de parentesco amplio, y a menudo, difusos. Pero que no por ello le restan autenticidad en el sistema de relaciones, unión y solidariad en momento de dificultades. Se basan, al menos de forma presunta, en una conceptualización que tendrían que ver más con el reconocimiento de un mismo linaje u origen, si bien este hecho en el presente puede verse sometido a juicio ${ }^{53}$.

e. La forma de dirigirse dentro del clan entre los miembros de éste es por medio del rol de parentesco que tienen. Pero siempre se utiliza el nombre kamba relacionado con el nacimiento, al que hacíamos alusión en el apartado anterior. De ahí la relevancia que posee en el ámbito de las relaciones familiares, tanto directas como extensas.

50 G. Wijeyewardene, “Definition, Innovation and History”, en G. Wijeyewardene (ed.): Ethnic Groups Across National Boundaries in Mainland Southeast Asia. Singapur: Institute of Southeast Asian Studies, 1990, pp. 1-13

51 C. H. Cerquera-González, “¿Clanes territoriales o clanes dispersos? Algunas consideraciones generales sobre la estructura social Wayúu”, en Jangwa Pana, vol. 7, n. ${ }^{1}$ (2008), pp. 58-69. J. A., Martínez, Introducción histórica a la antropología del parentesco. Madrid: Editorial Universitaria Ramón Areces, 2008.

52 Á. Díaz de Rada, “¿Dónde está la frontera? Prejuicios de campo y problemas de escala en la estructuración étnica en Sápmi”, en Revista de dialectología y tradiciones populares, vol. 63, n. 1 (2008), p. 201.

53 G. O. Alvarez, "Pós-dradivianoSateré-Mawé: parentesco y rituales de afinabiliadad”, en Vibrant: Virtual Brazilian Anthropology, vol. 8, n. ${ }^{\circ}$ (2011), pp. 375-402. E. P. Gené, "La impronta de Claude Lévi-Strauss en la antropología del parentesco", en El Genio Maligno: revista de humanidades y ciencias sociales, vol. 4, n. 2 (2009), pp. 1-12. 
Tabla 6. Denominación de distintos clanes akamba

\begin{tabular}{ll} 
Atui & Asii \\
Amutei & Akiimi \\
Atwanga & Aewani \\
Akitondo & Aombe \\
Aethnaga & Anzauni \\
Aiini & Akitutu \\
\hline
\end{tabular}

Fuente: elaboración propia

Una de las cuestiones sobre las que se solicitó información a la comunidad, en conexión directa con los modos de relación y la denominación fue la cuestión de la poligamia. Determinadas familias, al parecer, aún practican la poligamia, que ha sido una práctica muy extendida entre los kamba hasta la época de la colonización, puesto que "polygamy among the Akamba was a sign of social prestige and wealth" 54 . En algunos clanes, de hecho, es algo que se ha mantenido a pesar de la imposición del cristianismo. No obstante, esta costumbre no parece gozar de gran prestigio, al menos, en lo que se refiere a su práctica descubierta. Sí se muestra como más frecuente y mejor integrada desde la aceptación social, la poligamia no institucionalizada, bien por medio de la práctica de tener una esposa oficial y diversas concubinas; o bien por medio de relaciones extramatrimoniales. La tendencia, por lo que se muestra desde la observación, es hacia una reducción considerable de esta costumbre.

\section{Conclusiones}

Proceder a un acercamiento a un grupo étnico, con toda la problemática de este concepto que ya hemos indicado con anterioridad, que a priori resulta alejado y diferente a la perspectiva que podemos tomar como observadores, no se halla extensa de dificultades y limitaciones. Primeramente, debemos precisar las ideas de grupo y de etnia, se hallan en los lindes de la generalización que pueden dar lugar a las pretensiones holísticas de las que tratamos de huir, pero con las que a menudo nos topamos y nos dejamos seducir. No se puede dejar de lado, siguiendo a Díaz de Rada (2003) que "estas diversas imágenes de totalidad muestran hasta qué punto la aspiración metodológica del holismo está determinada por los modelos teóricos y morales de la cultura" (p. 237). No obstante, del encuentro y la convivencia con la familia kamba en el ámbito rural y en las relaciones diarias,

54 M. P. Kyalo, "Family values and rituals in changing cultural context: Analysis on traditional Akamba marriage and its implications for Christian marriage", en International Review of Social Sciences and Humanities, vol. 1 (2011), p. 74. 
hemos podido observar diversos aspectos que nos permiten, al menos, acercarnos a su vivencia y comprensión de las relaciones humanas y de los modos de relación con el entorno. A fin de cuentas, "la alteridad radical no es sino una ficción improductiva" y "el valor de las personas de nuestro campo no radica en ser otros, sino sencillamente en que son seres humanos" (Díaz de Rada, 2008, p. 202). Partiendo de esta premisa, que llevó a la reconstrucción significativa del trabajo de campo original, se ha pretendido, a raíz de la forma de denominarse a nivel de familia, y de los nombres kamba dados a la hora de nacer, comprender algunos elementos relacionados con las relaciones, el papel de los miembros de la familia, y sus nexos de conexión.

De ahí comprendemos, tras la observación, que la familia constituye uno de los aspectos constitutivos más destacados en el contexto de la tradición kamba de la zona rural de Mwuala, constituyendo, junto a la forma de relación, nombres y vehículo de comunicación, parte inherente de su patrimonio intangible. No se puede negar, no obstante, que este conjunto de tradiciones, como puede parecer lógico, ha estado sometido a un continuo y constante proceso de cambio, transformación, incorporación de elementos nuevos; pero al mismo tiempo, en cierne. En relación con esta consideración, cabría señalar el dinamismo de la realidad, que conlleva que el análisis cultural sea, de igual manera, dinámico. Este hecho se constata en los distintos influjos e interfecundación mutuos de los diferentes colectivos, que se basa en "los movimientos de la población al encuentro y el diálogo, el cambio de las culturas en el espacio y en el tiempo" 55 (Rössler, 2006, p. 20). Por ello, la observación y el análisis de la familia kamba, en el contexto de su comunidad, nos permite, siguiendo a Clifford (1986) una "mirada oblicua", por medio del "método dialogal" (p. 45). De esta forma, el conocimiento de la estructura y los modos de relación, aunque condicionados por la perspectiva del investigador, se pone en relieve la negación de la coetaneidad ("denialofcoevalness"56). La descripción que hemos ofrecido se basa en un momento determinado, en un espacio concreto, y con los condicionantes que responden a una evolución diacrónica, condicionada por diversos factores internos y externos que han conllevado que los kamba hayan adquirido, en el contexto de la actual localidad de Mwuala, las características que presentan hoy. En cualquier caso, el contacto con la comunidad de Mbuini ha permitido conocer muchos elementos que se convierten en lo que son los akamba de la actualidad, al menos en este momento. El contraste con otros kamba, que viven en contacto con otros grupos distintos, o que viven

55 M. Rössler, "Los paisajes culturales y la Convención del Patrimonio Mundial Cultural y Natural: resultados de reuniones temáticas previas", en E. Mujica Barreda (ed.): Paisajes culturales en Los Andes. Memoria narrativa, casos de estudio, conclusiones y recomendaciones de la reunión de expertos. Arequipa/Chivay, Lima, UNESCO, 2006, p. 20.

56 J. Fabian, Time and the Other. How Anthropology Makes Its Object. NuevaYork: Columbia University Press, p. 28. 
en grandes ciudades como Nairobi, conlleva a considerar que hay elementos en común con los que los kamba se sienten identificados. De ahí que, el uso de la lengua materna vernácula, la observación de determinados principios y, en especial, la autodefinición y autoidentificación con este colectivo, también denominado por los propios kenianos como tribu, se tornan en los aspectos básicos de su patrimonio y cultura viva, y por ende, de su identidad. De todos modos, el contacto con estos kamba que se hallan en la distancia permite conocer elementos de convergencia y de divergencia con el constructo kamba en sí mismo. Lugar y espacio y su conceptualización y definición en un mundo globalizado se tornan en una de las cuestiones clave para la comprensión de la situación global, de los elementos constitutivos de identificación en el controvertido dilema de los límites, imprecisos y cada vez más indefinidos entre lo regional y lo local. De ahí que Tsing manifieste que "wemight stop making a distinction between "global" forces and "local" places", puesto que a pesar de ser muy seductora, no resulta sino una falacia que no se puede adecuar a la realidad, trazando "globalist fantasies" (Tsing, 2000, p. 352). De ahí que los elementos que aportamos a lo largo del presente análisis den sólo pinceladas parciales de una colectividad dinámica y en constante proceso de transformación, muy a pesar de su percepción de estatismo relativo y de características definidas.

En cualquier caso, desde la convivencia con los kamba de Mwuala-Mbuini se han podido extraer una serie de modos de relacionarse inter e intra familiares que imponen unas expectativas comunitarias a las que el grupo espera que la persona responda de manera positiva. 
